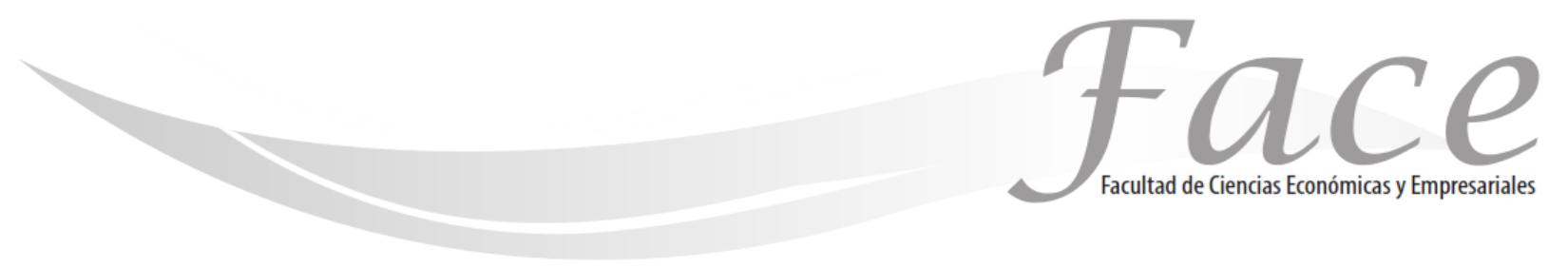

ISSN 1794-9920

Volumen $15-\mathrm{N}^{\circ} 2$

Año 2015

Págs. 29 - 37

\title{
LA FACTIBILIDAD DE LA COMERCIALIZACIÓN DE PRODUCTOS DE REALIDAD AUMENTADA PARA LA OPTIMIZACIÓN DEL PROCESO EDUCATIVO A NIVEL PRIMARIA EN LA ZONA NORTE DEL ESTADO DE VERACRUZ, MÉXICO *
}

\author{
Gwendolyne Barrera Silva** \\ Gil Santana Esparza*** \\ Manuel Cornelio Torres Maza***
}

Fecha de Recepción: 26 de Mayo 2015

Fecha de Aprobación: 29 de Agosto 2015

\begin{abstract}
Resumen:
El propósito de este artículo es analizar las oportunidades que tienen las novedades tecnológicas en el proceso educativo, proporcionando nuevas herramientas para mejorar la calidad de la educación y a partir de esta factibilidad buscar la comercialización de estos productos innovadores, proponiendo la herramienta educativa del futuro. Lo anterior genera un espacio de oportunidades para el desarrollo y comercialización de productos y proyectos como el Gestor de Realidad Aumentada en la Educación que permite combinar en tiempo real la realidad física con la realidad virtual en tres dimensiones, con elementos 3D que permiten aprender y entender mejor los contenidos académicos. El estudio es de tipo descriptivo, analítico a partir de los resultados de las investigaciones y factibilidad de los productos de la empresa.
\end{abstract}

Palabras clave: Factibilidad, comercialización, Realidad aumentada, tres dimensiones, realidad virtual, habilidades tecnológicas, educación.

\footnotetext{
* Artículo resultado del Proyecto de Investigación "Optimización del Proceso Educativo con técnicas de Realidad Aumentada", dentro del Instituto Tecnológico Superior de Pánuco, el cual se encuentra en la fase de prototipo tecnológico funcional, iniciado el 2 de febrero de 2015 y finalizada la primera etapa 11 de junio de 2015.

** Licenciada en Educación, docente de la carrera de Ingeniería en Gestión Empresarial del Instituto Tecnológico Superior de Pánuco. Correo: gwendybarrera@hotmail.com

***Ingeniero en Sistemas Computacionales, Maestro en Comunicación Académica, docente de la carrera de Ingeniería en Sistemas Computacionales del Instituto Tecnológico Superior de Pánuco. Correo: gil.santana@itspanuco.edu.mx

${ }^{* * \star *}$ Contador Público, Maestro en Ciencias Administrativas con especialidad en Relaciones Industriales, docente de la carrera de Contador Público del Instituto Tecnológico Superior de Pánuco. Correo: manuel_torres_maza@hotmail.com
} 


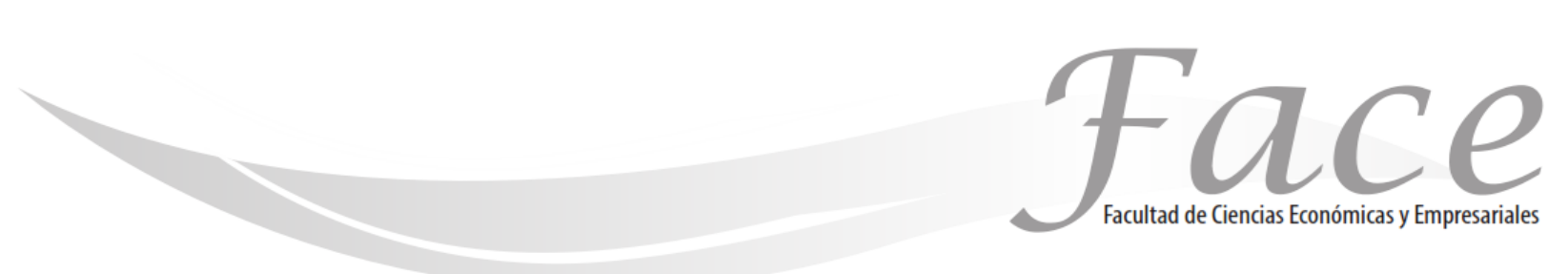

\title{
THE FEASIBILITY OF THE MARKETING OF AUGMENTED REALITY TO BENEFIT THE EDUCATIONAL PROCESS A PRIMARY LEVEL IN THE NORTH STATE OF VERACRUZ, MEXICO *
}

\begin{abstract}
:
The purpose of this article is to analyze the opportunities for technological innovations in the educational process, providing new tools to improve the quality of education and from this feasibility seek marketing of these innovative products, offering the educational tool of the future. This creates a window of opportunity for the development and marketing of products and projects as Manager Augmented Reality in Education that combines real-time physical reality with virtual reality in three dimensions with 3D elements that allow you to learn and understand better academic content. The study is descriptive, analytic from the results of investigations and feasibility of the company products.
\end{abstract}

Keywords: Feasibility, marketing, augmented reality, three-dimensional, virtual reality technology skills, education.

\section{A VIABILIDADE DO MARKETING DA REALIDADE AUMENTADA PARA BENEFICIAR O PROCESSO EDUCATIVO NÍVEL PRIMÁRIO NO ESTADO NORTE DE VERACRUZ, MÉXICO}

\section{Resumo:}

O objetivo deste artigo é analisar as oportunidades para inovações tecnológicas no processo educacional, fornecendo novas ferramentas para melhorar a qualidade do ensino ea partir deste viabilidade buscar comercialização destes produtos inovadores , oferecendo a ferramenta educacional do futuro. Isso cria uma janela de oportunidade para o desenvolvimento e comercialização de produtos e projetos como Gerente de Realidade Aumentada em Educação que combina realidade física em tempo real com realidade virtual em três dimensões com os elementos 3D que permitem que você aprender e entender melhor conteúdo acadêmico . O estudo é descritivo, analítico dos resultados das investigações e viabilidade dos produtos da empresa.

Palavras-chave: viabilidade, comercialização, realidade aumentada, tridimensionais, habilidades tecnologia de realidade virtual, educação. 


\section{INTRODUCCIÓN:}

A nivel internacional los organismos como la UNESCO han hecho énfasis en el uso de las Tecnologías de la Información y Comunicación como un medio para fortalecer los sistemas educativos y la economía de las regiones.

Así mismo, la educación en México ha ido evolucionando a través de las generaciones buscando mejorar las herramientas de enseñanza aprendizaje en las aulas, auxiliándose con tecnologías innovadoras como la realidad aumentada, la cual es un área que no ha sido explotada en la educación como otras áreas de la tecnología.

Al mismo tiempo, la Secretaría de Educación Pública en México ha incorporado como estrategia en las instituciones educativas la incorporación del enfoque basado en competencias acompañado por corrientes constructivistas en pro de la excelencia académica y personal del alumno.

En base a lo anterior, en el presente trabajo se propuso estudiar la aplicación de las herramientas didácticas basadas en la realidad aumentada con el fin mismo de impulsar la innovación en las aulas y acercar, a bajo costo, esta tecnología a las instituciones educativas de nivel básico. En el estudio se muestra que las instituciones educativas en la zona norte del estado de Veracruz cuentan con centros de cómputo, y sin embargo los docentes no utilizan la computadora como herramienta en sus estrategias de enseñanza aprendizaje. Se muestra, también, que los motivos por los cuales no se utiliza el centro de cómputo son básicamente, porque no existe material didáctico audiovisual y ello trae como consecuencia que el alumno no tenga acceso continuo a las nuevas tecnologías.

Lo anterior ha presentado un espacio de oportunidades para el desarrollo y comercialización de productos de software derivados de proyectos como el Gestor de Realidad Aumentada en la Educación que fue diseñado por un equipo de investigadores del Instituto Tecnológico Superior de Pánuco. Este proyecto ha permitido combinar en tiempo real la realidad física con la realidad virtual en tres dimensiones, para desarrollar una experiencia denominada Realidad Aumentada que en sus pruebas piloto ha propiciado el fortalecimiento de competencias en los alumnos potenciando el entorno físico con elementos $3 \mathrm{D}$ que permiten aprender y entender mejor los contenidos académicos.
También se presenta el análisis del mercado potencial, la demanda y oportunidades que se tienen, finalizando con el proceso de servicio y comercialización que se ofrece como empresa de productos software de realidad aumentada a los clientes potenciales en la región de la zona norte de Veracruz.

\section{MARCO TEÓRICO:}

El planteamiento de la idea de comercialización de productos de software basados en la realidad aumentada ha hecho necesario analizar los referentes teóricos necesarios para definir la propuesta de valor que transforme la actividad académica tradicional en una experiencia visual interactiva para el fortalecimiento de competencias en los alumnos, pero con la firme intención de hacer llegar la propuesta a precios accesibles a los clientes y al mismo tiempo que sea rentable para la empresa. Los referentes teóricos conceptuales analizados y estudiados se mencionan a continuación.

Como punto de partida es importante analizar a profundidad las implicaciones del concepto de Marketing ya que, como lo menciona Ferrell (2011), de acuerdo a los cambios en el concepto por la American Marketing Association (AMA), Marketing se define como el proceso de planear y ejecutar la idea, la fijación de precios, la promoción y la distribución de ideas, bienes y servicios para crear intercambios que satisfagan los objetivos individuales y organizacionales. Lo anterior es importante de considerar en el presente proyecto que pretende comercializar productos software que requieren un gran trabajo de promoción para ser incorporados con éxito en nuevos mercados.

Al igual que lo anterior se debe considerar y tomar en cuenta las herramientas denominadas 4P's que Kotler (2001) en su obra las define como las herramientas de mercadotecnia que utiliza la empresa para alcanzar sus objetivos en el mercado meta, las cuales puntualiza en el concepto de cada una:

Producto: representa la oferta tangible de la firma al mercado, incluyendo calidad, diseño, características, marca y el empaque del producto. Herramienta más fundamental de la mercadotecnia.

Precio: la cantidad de dinero que los clientes tienen que pagar por el producto. Herramienta crítica de la mercadotecnia. 
Plaza o colocación: comprende las diferentes actividades que la empresa emprende para que el producto sea accesible y esté a disposición de los consumidores meta. Promoción: diferentes actividades que la empresa realiza para comunicar las excelencias de sus productos $y$ persuadir a los clientes objetivos para su adquisición.

Además de lo anterior, es importante considerar los avances tecnológicos, tal y como lo menciona Ferrel (2011) argumentando que las tecnología se han convertido en uno de los productos más atractivos para el comercio, afirmando también, que esta tecnología se refiere a la forma en que logramos tareas específicas o los procesos que usamos para crear las "cosas" que consideramos nuevas. De todas las nuevas tecnologías generadas en los últimos 30 años, ninguna ha tenido un mayor impacto en marketing que los avances en la tecnología de cómputo y de información, la cual ha cambiado la forma en que viven los consumidores y empleados y en que los mercadólogos operan para satisfacer sus necesidades.

También ha sido importante en este trabajo tomar en consideración las necesidades de la educación, ya que en el Plan de Educación Primaria se hace mención a las Tecnologías de la Información y la Comunicación (TIC) las cuales menciona que son fundamentales para el desarrollo económico, político y social de los países, y cobran sentido ante la existencia de la economía del conocimiento (SEP, 2011). Se ha comprobado que la ausencia de una política de tecnologías de la información y la comunicación en la escuela pública aumenta la desigualdad entre las personas.

Derivado de lo anterior, se hace necesario considerar, también, las transformaciones en la enseñanza de las ciencias, ya que, la naturaleza de la ciencia contempla la construcción del conocimiento científico, los procesos del desarrollo de la ciencia, los entramados sociales de las comunidades científicas y los procesos implícitos en la construcción del conocimiento. La relevancia que este campo ha adquirido se basa en el supuesto de que una comprensión clara de cómo se construye la ciencia tiene implicaciones importantes para mejorar la educación en ciencias. Durante la primera revolución en la enseñanza de las ciencias las ideas del psicólogo Bruner tuvieron una importante influencia. La meta educativa que compartían estas iniciativas fue la enseñanza del conocimiento científico a través de los métodos de la ciencia. La segunda revolución estuvo acompañada de diversos materiales para mejorar la educación en ciencias naturales, como la incorporación de la tecnología educativa (Flores-Camacho, 2012).

\section{METODOLOGÍA:}

El presente trabajo se ha desarrollado aplicando las características del método científico para que sea congruente, confiable y fundamentado en las teorías relativas al objeto de estudio, ya que se requiere que los resultados tengan el grado máximo de confiabilidad. Se ha realizado una investigación documental combinada con una investigación de campo apuntando hacia la innovación en la comercialización de productos de software basados en realidad aumentada. Se ha definido una secuencia de pasos planeados de antemano para asegurar que los datos que se obtengan permitan un análisis objetivo que lleve a conclusiones válidas. Para documentar el análisis situacional se ha recopilado información mediante entrevistas y cuestionarios a profesores y directivos de las instituciones de educación básica de la zona norte de Veracruz. Se ha estado trabajado en el análisis situacional desde el mes de enero del 2015 y continúa en desarrollo y fortalecimiento.

\subsection{Situación problemática que se puede resolver.}

El análisis situacional se llevó a cabo en la zona de Pánuco, Veracruz, donde se ubican 124 instituciones educativas de nivel primaria, para lo cual se aplicó la siguiente fórmula para determinar el tamaño de la muestra a entrevistar.

\begin{tabular}{|c|c|}
\hline & $N \sigma^{2} Z^{2}$ \\
\hline Dónde: & $n=$ \\
\hline $\mathbf{N}=124$ & $(N-1) e^{2}+\sigma^{2} Z^{2}$ \\
\hline \multicolumn{2}{|l|}{$\sigma=0.5$} \\
\hline $\mathbf{Z}=1.96$ & $(124)(0.5)^{2}(1.96)^{2}$ \\
\hline$e=0.05$ & $(124-1)(0.05)^{2}+(0.5)^{2}(1.96)^{2}$ \\
\hline
\end{tabular}

Al aplicar la fórmula se obtiene una muestra $n=94$, a la cual se entrevistó obteniéndose, entre otros, los resultados que a continuación se describen. 


\section{RESULTADOS:}

A continuación se describen los datos significativos obtenidos de las encuestas aplicadas, donde se detecta la necesidad de productos nuevos e innovadores en la enseñanza y orientados a las generaciones actuales.

La mayoría de las instituciones educativas de nivel primaria tienen instalaciones de centros de cómputo para trabajo de los alumnos.

Este dato es importante porque indica la existencia de computadoras que al ser parte del activo fijo vigente en las instituciones educativas, éstas ya no requieren inversiones adicionales y el producto software a ofertar tendría la infraestructura necesaria para ser instalado.

Figura $\mathrm{N}^{\circ} 1$.

Existencia de equipos de cómputo en las instituciones educativas.

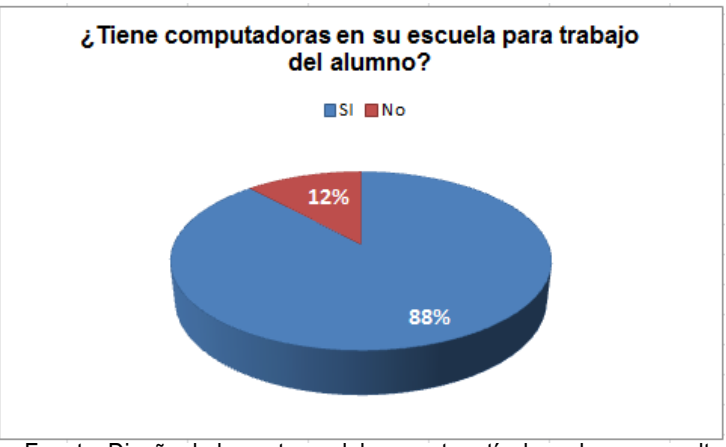

Fuente: Diseño de los autores del presente artículo en base a resultados de encuestas.

Sin embargo se detectó que no todos los docentes utilizan la computadora como herramienta en sus estrategias de enseñanza aprendizaje.

Este dato nos revela un área de oportunidad para que el docente explote el equipo de cómputo existente en las instituciones aplicando el producto software de realidad aumentada.
Figura $\mathrm{N}^{\circ} 2$.

Porcentaje de docentes que utiliza la computadora para clases.

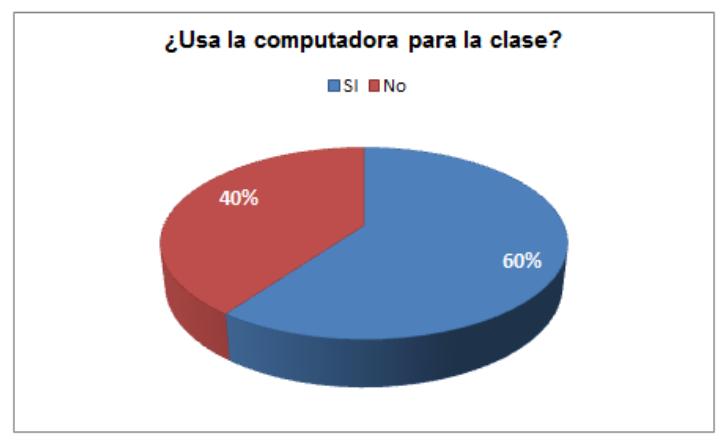

Fuente: Diseño de los autores del presente artículo en base a resultados de encuestas.

Los motivos por los cuales no se utiliza el centro de cómputo son básicamente dos: no existe material didáctico audiovisual y se carece de maestros de computación.

En este punto se pretende reforzar el material didáctico del docente con las herramientas de software de realidad aumentada. Al ser material de realidad aumentada en base a la necesidad propia de cada docente y de cada asignatura, se garantiza un producto software que será utilizado de manera efectiva en las estrategias de enseñanza aprendizaje. El servicio que se ofrece y el producto son intuitivos y amigables por lo que no requiere especialistas para ponerlo en funcionamiento.

Figura $\mathrm{N}^{\circ} 3$.

Motivos por los cuales no se utiliza la computadora para clase.

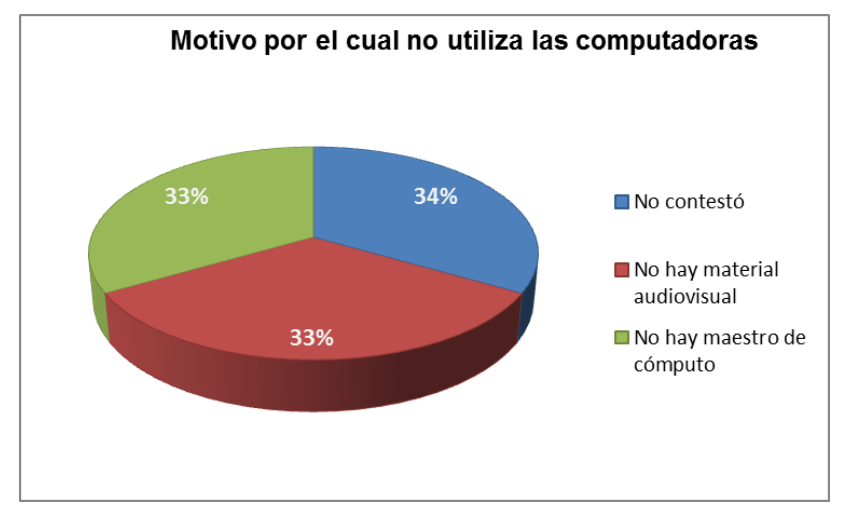

Fuente: Diseño de los autores del presente artículo en base a resultados de encuestas. 
Figura $\mathrm{N}^{\circ} 5$.

El punto anterior trae como consecuencia que el alumno no tenga acceso continuo a esta tecnología.

Con la aplicación de las herramientas de software de realidad aumentada en base a necesidad de asignatura y docente se garantiza que el alumno interactúe en forma contínua con las nuevas tecnologías.

Figura $\mathrm{N}^{\circ} 4$.

Frecuencia de interacción del alumno con la computadora.

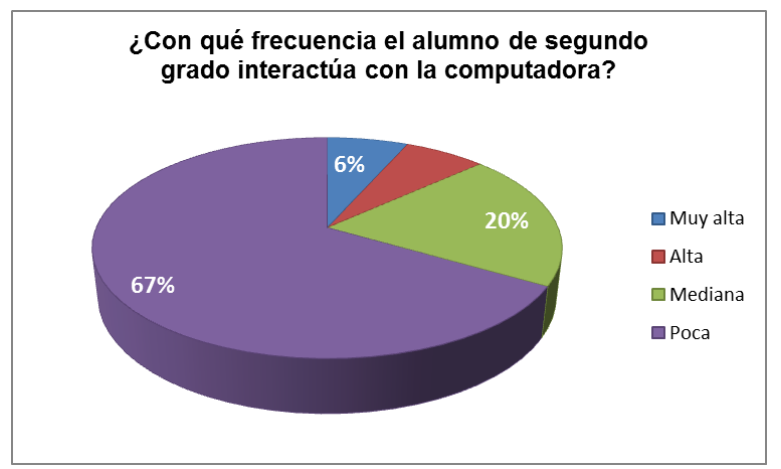

Fuente: Diseño de los autores del presente artículo en base a resultados de encuestas.

Sin embargo, el docente considera que la computadora es importante como herramienta de enseñanza aprendizaje y que podría utilizarse para complementar las materias de conocimiento del medio, español y matemáticas principalmente.

En este punto apreciamos que el docente tiene interés en utilizar la tecnología como apoyo en sus asignaturas, por lo cual, se pretende aprovechar la disposición y apertura para posicionar el producto software de realidad aumentada como tecnología novedosa y actual.
Materias en las cuales se considera importante el uso de la computadora como herramienta de enseñanza aprendizaje.

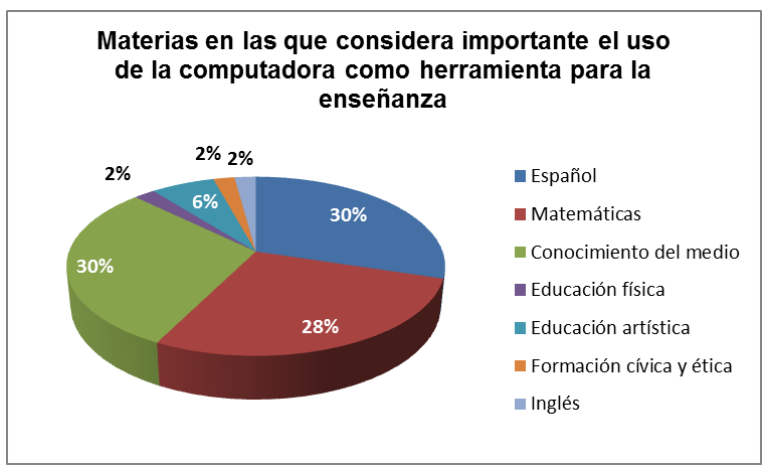

Fuente: Diseño de los autores del presente artículo en base a resultados de encuestas.

A lo anterior se agrega que los docentes han detectado que la mayoría de los alumnos son visuales y que requieren materiales que beneficien esa característica.

Parte importante en este trabajo es el hecho de comercializar productos de software que benefician el estilo de aprendizaje visual y auditivo que las generaciones actuales tienen muy desarrolladas.

Figura $\mathrm{N}^{\circ} 6$

Tipos de alumnos.

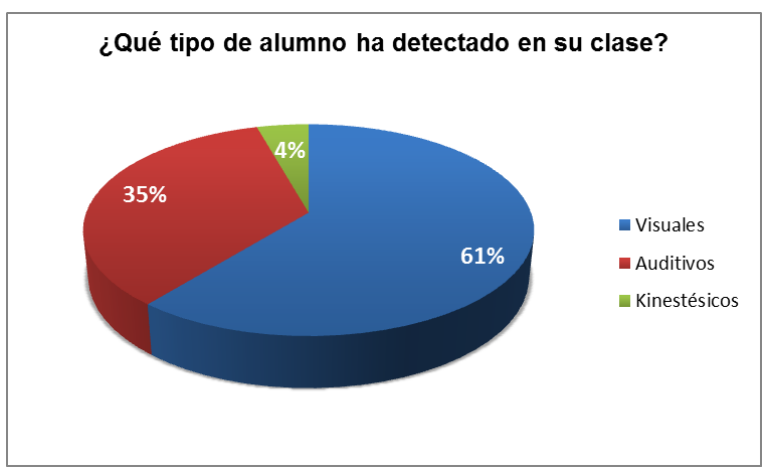

Fuente: Diseño de los autores del presente artículo en base a resultados de encuestas.

La mayoría del software que se maneja es el tradicional administrativo como Office, Enciclomedia, Encarta y en algunos casos visualización de video en Youtube. El software actualmente utilizado tiene elementos importantes y útiles para impartir la clase por parte del docente, sin embargo, no aportan soluciones directas que beneficien los temas de interés en base a la necesidad del alumno. 
Figura $\mathrm{N}^{\circ} 7$.

Software utilizado con más frecuencia.

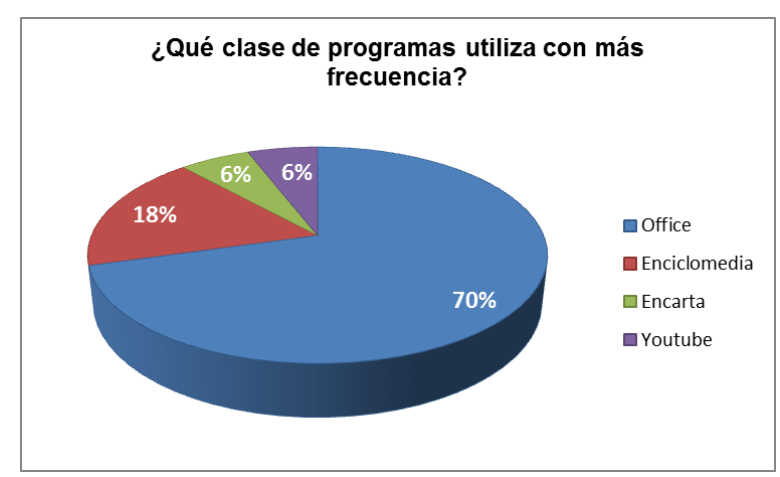

Fuente: Diseño de los autores del presente artículo en base a resultados de encuestas.

Por lo anterior, se puede concluir que existen necesidades de soluciones directas, atractivas e innovadoras que van dirigidas a los alumnos de las generaciones actuales que en su mayoría son visuales y auditivos para beneficiar las actividades de enseñanza-aprendizaje entre docente y alumno que propicien el aprendizaje significativo y asimilación de contenidos académicos con el uso de las tecnologías de la información y la comunicación, creando una oportunidad de comercialización donde la empresa OPERA puede encontrar un amplio mercado potencial dentro de la educación.

\subsection{Mercado Potencial}

Como una primera etapa la empresa OPERA está dirigida a las 124 instituciones educativas de nivel primaria de la zona de Pánuco, Ver. Extendiéndose en etapas posteriores a la zona norte de Veracruz y zona sur de Tamaulipas, buscando como meta final todas las instituciones educativas públicas y privadas de nivel primaria del país.

\subsection{Demanda y oportunidades del mercado.}

En base al análisis situacional la empresa OPERA tendría demanda y oportunidad de aceptación en el mercado potencial considerado, ya que el $100 \%$ de los docentes encuestados estaría a favor de que la institución adquiriera productos de realidad aumentada para apoyo en sus clases.
Figura $\mathrm{N}^{\circ} 8$.

Oportunidad de mercado de la empresa OPERA.

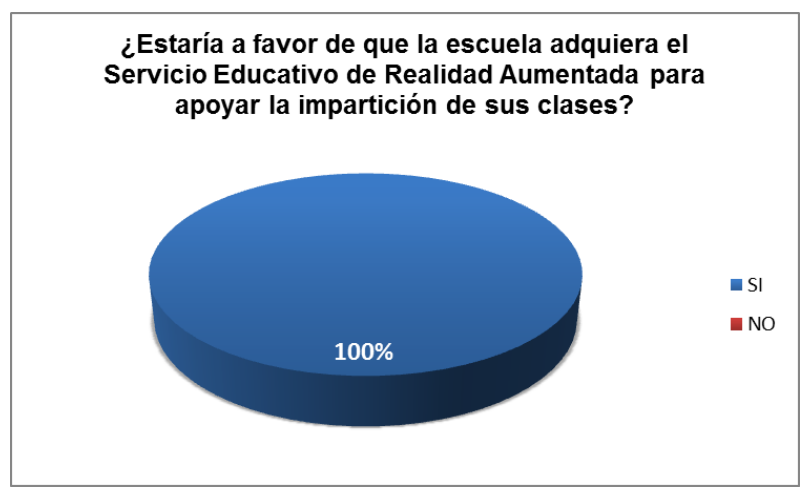

Fuente: Diseño de los autores del presente artículo en base a resultados de encuestas.

\section{DISCUSIÓN:}

\subsection{Compañía}

Como resultado de la investigación anterior se ha creado la empresa OPERA la cual está conformada por un grupo de profesionales del área de Sistemas Computacionales y del área de Gestión Empresarial que garantizan al cliente la calidad en el uso de tecnologías de vanguardia que dan como resultado final la Optimización del Proceso Educativo con técnicas de Realidad Aumentada (OPERA).

En OPERA se cree y se practica como una forma de vida la calidad, la visión de futuro y el adecuado uso de la creatividad en la forma de trascender en la educación. La filosofía de la compañia respalda la calidad del proceso de acompañamiento que se proporciona a los clientes. Se está a favor de la innovación y creatividad, calidad, respeto, desarrollo integral y excelencia del personal. El equipo colaborativo está conformado por hombres y mujeres motivados y competentes que se abren paso hacia la mejora continua con la intención de servir con energía y con un alto compromiso en el logro de las metas.

\subsection{Promoción.}

Para llegar a los clientes se aplicarán canales de información y canales de compra estratégicos a través de los cuales los clientes conocerán y/o comprarán el software de acompañamiento del proceso educativo ofrecido por OPERA.

En esta primera etapa el canal utilizado será la promoción directa con los clientes potenciales a través de puntos de venta con folletos informativos y demostraciones en sitio. 
ISSN: 1794-9920 Julio - Diciembre 2015

Volumen 15 Número 2 Año 2015 Págs. 29 - 37

Para etapas posteriores se diseñará el sitio web OPERA y se establecerán alianzas estratégicas con distribuidores de software educativo.

Figura $\mathrm{N}^{\circ} 9$

Diagrama del proceso OPERA
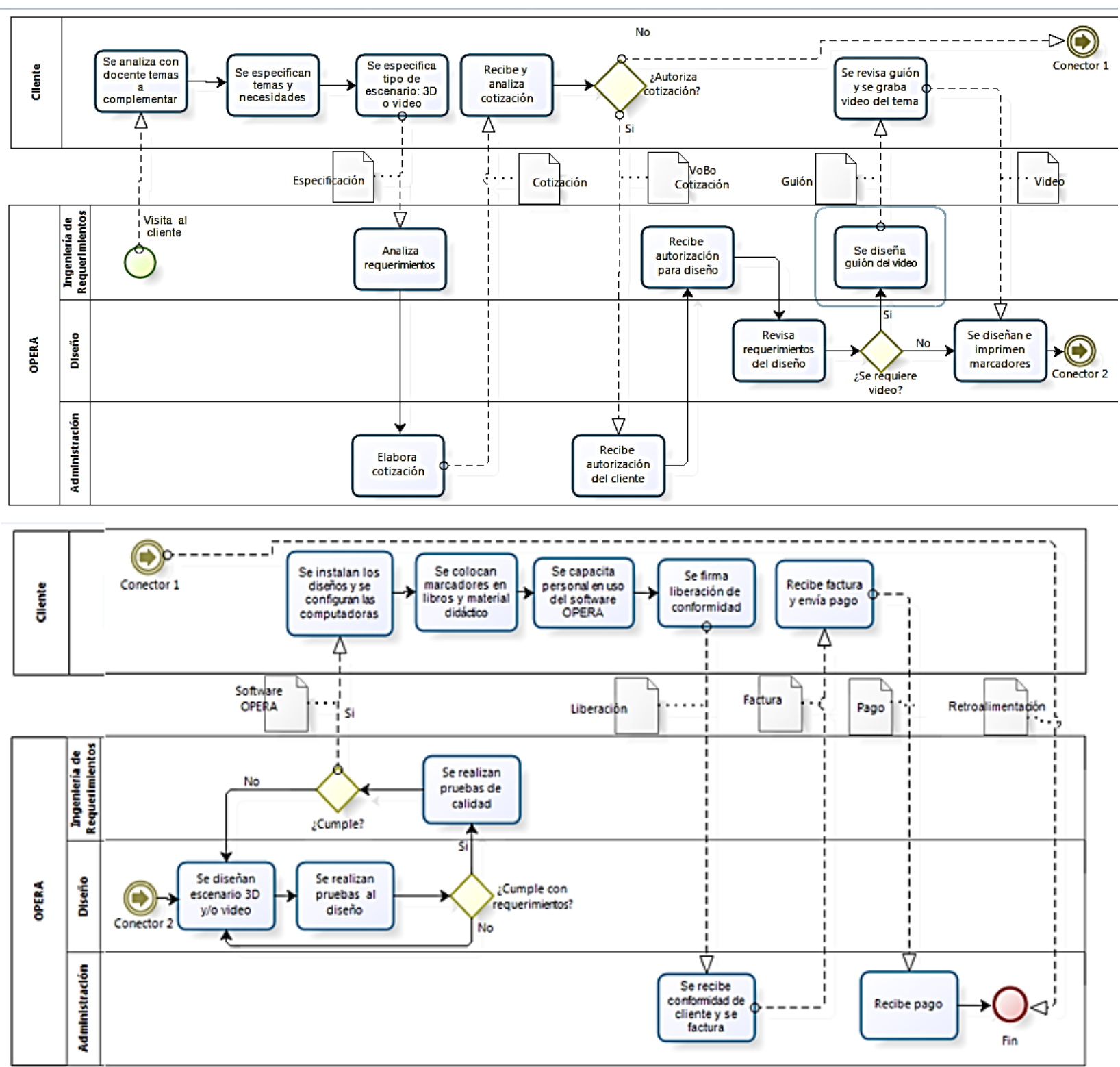

Fuente: Idea y diseño de los autores del presente artículo. 


\section{CONCLUSIONES:}

El software que se proporciona está diseñado para beneficio del cliente por lo cual se han tomado en cuenta los atributos funcionales, eficaces y psicológicos que el cliente va a percibir. Por lo tanto, se puede afirmar que la comercialización y correcta aplicación de software de realidad aumentada como herramienta didáctica tiene impacto en el ámbito académico ya que permite a los alumnos mejorar la comprensión en temas complejos o difíciles de asimilar. Así también, se tiene un impacto en el ámbito de las tecnologías ya que las instituciones pueden dar cumplimiento a la observación de organismos nacionales e internacionales que sugieren el uso de las nuevas tecnologías para el fortalecimiento de la economía y las regiones. $Y$ además, se genera impacto en el ámbito social al formar individuos con formación tecnológica que en la actual sociedad de la información y del conocimiento son parte activa en los procesos académicos, culturales y económicos del país.

Para cumplir con lo anterior, lo que ofrece la empresa es el acompañamiento en el proceso educativo mediante el diseño de contenidos didácticos combinando la realidad física con la realidad virtual en tres dimensiones para generar una experiencia denominada Realidad Aumentada que propicia y fortalece la asimilación de contenidos de los programas de estudio para beneficio de los actores involucrados: alumnos, docentes e institución.

\section{REFERENCIAS:}

Ferrell, O. C. y Hartline M. D. (2011). Estrategia de marketing, (Quinta edición). D.F. México: Cengage Learning, Inc

Flores-Camacho. (2012). La enseñanza de la ciencia en la educación básica en México. D.F., México: INEE.

González Morcillo, C. y Vallejo Fernández, D. (2011). Realidad aumentada un enfoque práctico con ARToolKit y Blender. (Primera edición). Madrid, España: Bubok Publishing S.L.
Kashi, A. E. (2012). ActionScript 3.0 para desarrolladores: programación orientada a objetos y buenas prácticas. (Primera edición). Buenos Aires, Argentina: Alfaomega.

Kotler, P. (2001). Dirección de Mercadotecnia Análisis, planeación, Implementación Y control. (Octava Edición). Evanston: Estados Unidos. Pearson Educación

SEP. (2011). Plan de Estudio 2011. Educación Básica. D.F., México: SEP.

SEP. (2011). Las ciencias naturales en educación básica: formación de ciudadanía para el siglo XXI. D.F., México: SEP. 